\title{
Chaining in enterococci revisited: correlation between chain length and gelatinase phenotype, and gelE and $f s r B$ genes among clinical isolates of Enterococcus faecalis
}

Enterococci, like streptococci, are typically described as showing chaining in Gramstains of clinical samples. The degree of chaining seems to vary amongst enterococcal clinical isolates; however, there are no recent studies validating chaining by enterococci since they were classified out of the genus Streptococcus nor any studies of the mechanism of cellular chain length. A marked increase in chaining seen in an Enterococcus faecalis OG1RF mutant (TX5128) has been explained by the lack of the GelE protease (gelatinase) suggesting the possibility that chaining among clinical isolates (one-third of which are gelatinase non-producers) might be associated with the absence of GelE (Singh et al., 2005; Waters et al., 2003). GelE is an enterococcal zinc-metalloprotease (Bleiweis \& Zimmerman, 1964) that is capable of degrading a broad spectrum of substrates, including insoluble collagen fragments (Makinen \& Makinen, 1994) and polymerized fibrin (Waters et al., 2003). Synthesis of GelE in E. faecalis is regulated by the $f$ sr locus (Qin et al., 2001), a quorum-sensing system that positively regulates expression of gelE. The $f s r$ locus and gelatinase production have been shown to contribute to the virulence of E. faecalis OG1RF in several models, including mouse peritonitis, Caenorhabditis elegans and biofilm formation (Mohamed et al., 2004; Qin et al., 1998, 2000; Sifri et al., 2002; Waters et al., 2003).

In the present study, a collection of clinical isolates of E. faecalis was studied to determine the degree of variation in the chaining phenotype and to establish whether the lack of GelE production or the absence of gelE or $f s r B$ genes among clinical isolates might explain any differences found. Since a correlation between chain length and disruption of gelE in the mutant TX5128 was demonstrated in vitro, such a correlation in clinical isolates might also be helpful to predict the absence/presence of important virulence factors (i.e. GelE) or their regulatory genes (i.e. $f_{s r B}$ ) with a simple clinical microbiology observation. Therefore, the number of cells per chain in isolates grown in broth was determined by light microscopy, and a correlation between chain length and genotype was attempted.

A total of 106 clinical isolates of E. faecalis [including blood (endocarditis and non-endocarditis), urine and community sources] from different geographical and clonal origins present in our collection and previously characterized for the presence of gelE and $f_{s} B$ and for the GelE phenotype (on Todd-Hewitt agar plates with $3 \%$ gelatin) (Roberts et al., 2004) was studied to determine the chaining phenotype (see below). E. faecalis strains OG1RF (wild-type, GelE ${ }^{+}$; Murray et al., 1993), TX5128 (a gelE disruption mutant derived from OG1RF, GelE ${ }^{-}$; Qin et al., 1998) and TX5266 (an $f s r B$ deletion mutant that is $\mathrm{GelE}^{-}$under routine conditions; Qin et al., 2001) were used as controls in the chaining experiments. All isolates were incubated in brain heart infusion broth overnight at $37^{\circ} \mathrm{C}$ with agitation (150-200 r.p.m.). Kanamycin $\left(2000 \mu \mathrm{g} \mathrm{ml}^{-1}\right)$ was added for TX5128 (gelE mutant) (Waters et al., 2003). The next day, the culture was diluted to obtain a turbidity equivalent to 0.5 McFarland $\left(A_{625} 0.08-0.10\right.$; $10^{8}$ c.f.u. $\mathrm{ml}^{-1}$ ) and then incubated for $5 \mathrm{~h}$ at $37^{\circ} \mathrm{C}$ with agitation (150-200 r.p.m.). Cells were harvested by centrifugation, resuspended in $5 \mathrm{ml} 0.9 \%$ saline and $5 \mu \mathrm{l}$ was spotted on a glass slide, Gram-stained, covered with a coverslip and visualized under a light microscope. To evaluate the chaining phenotype, each isolate was analysed and the number of cells per chain (a chain is defined as the presence of $\geqslant 1$ cell) in 50 randomly chosen chains at $\times 100$ magnification (usually four different power fields) was recorded. All isolates were evaluated in a blind manner by two observers (without knowing the origin, identification of the isolate, GelE phenotype or genotype). Discrepancies between observers were resolved by performing additional blind experiments until agreement was reached.

The following criteria were used to classify every isolate: an isolate was categorized as a short chainer if $\geqslant 80 \%$ of the chains (out of 50 assessed) had $\leqslant 4$ cells (and thus, $<20 \%$ had $>4$ cells); as a medium chainer if $41-79 \%$ of chains had $\leqslant 4$ cells; as a long chainer if $\leqslant 40 \%$ of chains contained $\leqslant 4$ cells; and as an extra-long chainer when $>70 \%$ of chains contained $\geqslant 10$ cells. Correlations between genotype/phenotype and chain length were performed for every category, with an initial analysis comparing short and non-short chainer (i.e. the sum of all medium, long and extra-long chainforming isolates) subgroups. A Fischer exact test was performed to establish statistically significant differences between short-chain-forming isolates and other categories; $P<0.05$ was considered significant.

There was a marked difference in the degree of chaining amongst clinical isolates, ranging from short chainers (Fig. 1a; $79 \%$ of isolates; range of the mean number of cells per chain per isolate, 1.0-3.5; mean number of cells per chain of all isolates, 2.3) to extra-long chainers (Fig. 1e; $3 \%$ of isolates; mean number of cells per chain per isolate, $>15$; mean number of cells per chain, 27). Amongst the remaining isolates, $13(12 \%)$ were classified as medium chainers (Fig. 1b, c; range of the mean number of cells per chain per isolate, $>3.5-7.5$; mean number of cells per chain of all isolates, 5.0) and 5 (4\%) were classified as long chainers (Fig. 1d; range of the mean number of cells per chain per isolate, $>7.5-15.0$; mean number of cells per chain, 11.3). Short-chaining isolates were independently compared to each of the other chaining categories with regard to 

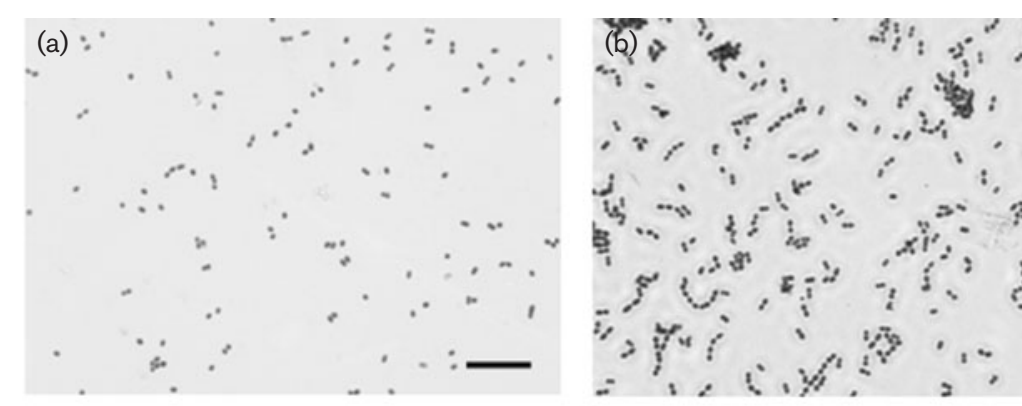

(e)

(d) $)$

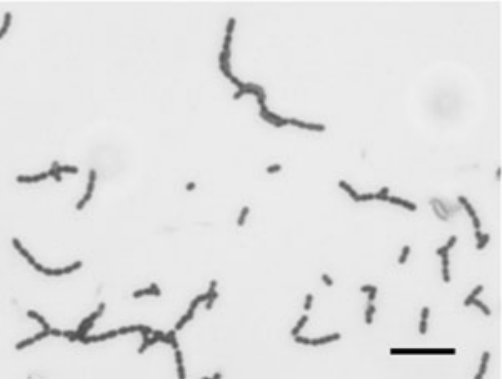

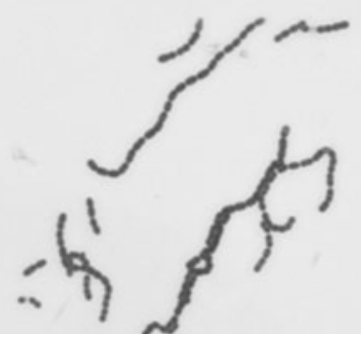
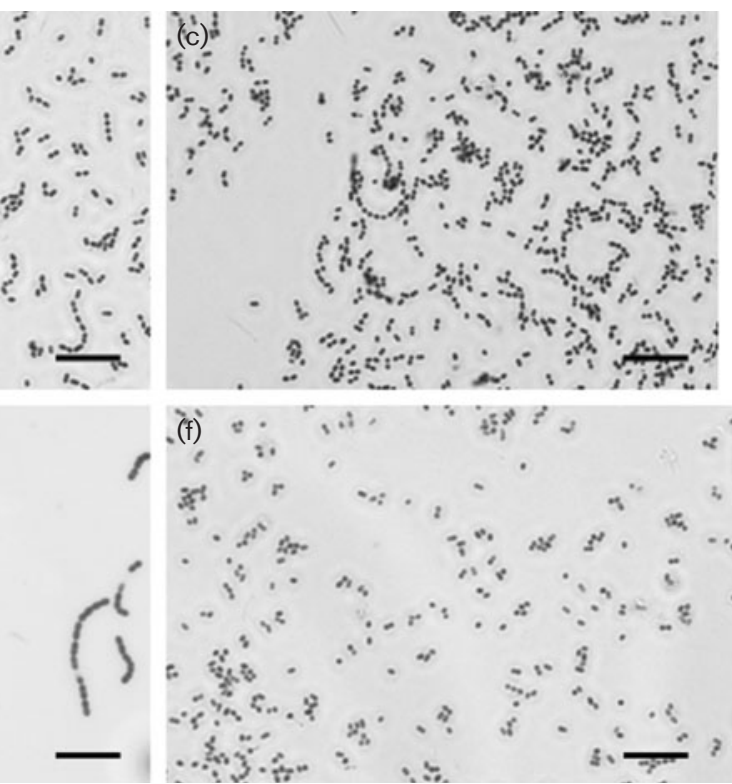

Fig. 1. E. faecalis chaining phenotypes. Gram stains of isolates after $5 \mathrm{~h}$ incubation (bar, $20 \mu \mathrm{m}$ ): (a) OG1RF (short chain phenotype); (b) TX5266 (fsrB mutant) and (c) TX5128 (gelE mutant) (medium chain phenotypes); (d) TX0621 (long chain phenotype), $\mathrm{Bla}(+)$; (e) TX0623 (extra-long chain phenotype), Bla(+); (f) V583, representative of BVE (Bla-Vancomycin'-endocarditis) clone, lacking $\beta$-lactamase (short chain phenotype).

the presence (or absence) of GelE, gelE and $f s r B$. A similar analysis was performed comparing short-chaining isolates and the sum of the other subgroups (medium, long and extra-long chainers). There was no correlation between the absence of $g e l E, f_{s r} B$ or gelatinase production and isolates with medium, long or extra-long chains (Table 1) or when non-short isolates (grouping isolates categorized as having either medium, long or extra-long chains) were compared to those with short chains (all $P>0.05$ ) (Table 1). Indeed, the majority of non-short isolates produced gelatinase and had the gelE or $f s r B$ gene: $68 \%$ for GelE, $95 \%$ for gelE and $77 \%$ for $f s r B$. Moreover, when long and extra-long chainers were grouped together, only 33, 11 and $22 \%$ lacked GelE, gelE and $f s r B$, respectively. A similar percentage of short chain formers lacked GelE (36\%), gelE $(12 \%)$ or $f s r B(28 \%)$. These results indicate that chaining among clinical isolates is not dependent on the lack of gelatinase, $f s r B$ or gelE. Under the conditions used in this study, E. faecalis OG1RF was classified as a short chainer $(\geqslant 80 \%$ of the chains had $\leqslant 4$ cells), whereas both TX5128 (gelE disruption mutant) and TX5266 ( $f_{s r} B$ deletion mutant) were categorized as medium chainers (mean number of cells per chain of all isolates, 5.0) (Fig. 1b, c).

The mechanism for chaining in E. faecalis strains is unknown. Purified gelatinase has been shown to activate the muramidase-1 autolysin and a relationship between gelatinase and autolysis has been proposed as a mechanism to regulate chain length (Shockman \& Cheney, 1969). However, neither gelatinase production nor a lack thereof correlated with chaining. It may be

Table 1. Chaining phenotype and gelE, $f s r B$ and GelE phenotypes of the $E$. faecalis strains studied

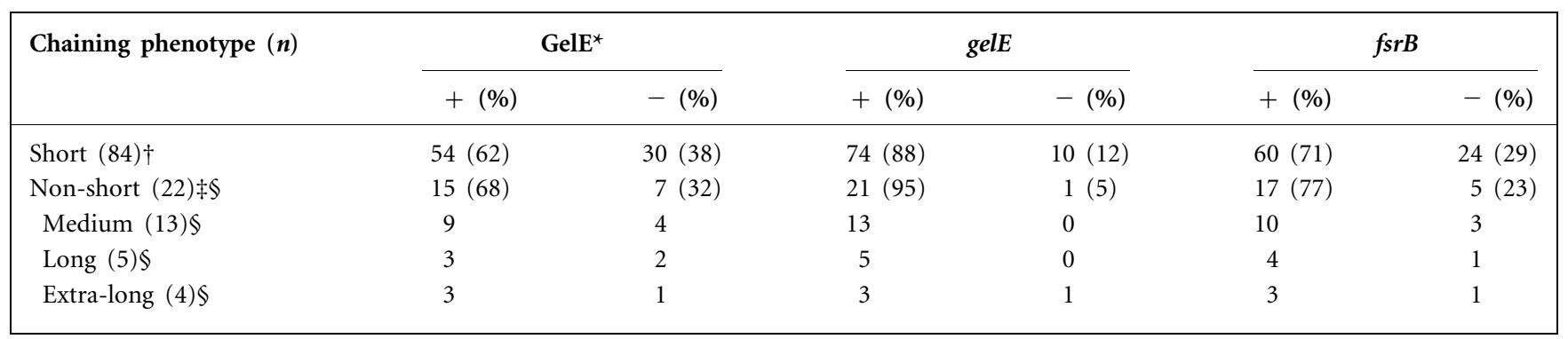

${ }^{\star}$ Refers to gelatinase assay on Todd-Hewitt agar with $3 \%$ gelatin.

$\dagger$ Indicates $\geqslant 80 \%$ of the chains had $\leqslant 4$ cells.

$\ddagger$ Category encompasses isolates with medium, long and extra-long chains (see text for definitions).

$\S$ All subgroups were compared to the short chainers for GelE, gelE and $f_{s} r B . P>0.05$ in all cases. 
that $\mathrm{GelE}^{-}$clinical isolates have developed a compensatory mechanism to replace the lack of gelatinase. It has also been shown (Nakayama et al., 2002) that most clinical isolates that display the $\mathrm{GelE}^{-}$phenotype lack a $23.9 \mathrm{~kb}$ region upstream of $f_{s r} C$, whereas TX5128 (gelE disruption mutant) still retains this region (Roberts et al., 2004). Whether the genes of this region activate other mechanisms that influence cell separation in clinical isolates is unknown.

In summary, marked variation in chaining was found in clinical isolates of E. faecalis. However, despite a pronounced effect of disruption of the gelE gene of strain OG1RF on chaining, neither the lack of GelE production nor the absence of the gelE gene was associated with chaining among clinical isolates, with some $\mathrm{GelE}^{+}$isolates showing marked chaining ( $>15$ cells per chain) and some $\mathrm{GelE}^{-}$and gelE-lacking isolates showing predominantly short chains $(\leqslant 4$ cells per chain).

\section{Acknowledgements}

This work was supported by NIH grant R37 AI47923 from the Division of Microbiology and Infectious Diseases to B. E. M. We are grateful to Natalia Mendoza, Universidad El Bosque, Colombia, for statistical analysis, and Kavindra Singh and Sreedhar Nallapareddy for helpful discussions in the preparation of this manuscript.

Cesar A. Arias, ${ }^{1,2,3}$ Leonardo Cortes ${ }^{3}$ and Barbara E. Murray 1,2,4

${ }^{1}$ Center for the Study of Emerging and Reemerging Pathogens, Division of Infectious Diseases, University of Texas Medical School at Houston, Houston, TX 77030, USA
${ }^{2}$ Department of Internal Medicine, University of Texas Medical School at Houston, Houston, TX 77030, USA

${ }^{3}$ Bacterial Molecular Genetics Unit, Universidad El Bosque, Bogota, Colombia

${ }^{4}$ Department of Microbiology Molecular Genetics, University of Texas Medical School at Houston, Houston, TX 77030, USA

Correspondence: Barbara E. Murray (bem.asst@uth.tmc.edu)

Bleiweis, A. S. \& Zimmerman, L. N. (1964). Properties of proteinase from Streptococcus faecalis var. liquefaciens. J Bacteriol 88, 653-659.

Makinen, P. I. \& Makinen, K. K. (1994). The Enterococcus faecalis extracellular metalloendopeptidase inactivates human endothelin at bonds involving hydrophobic amino acid residues. Biochem Biophys Res Commun 200, 981-985.

Mohamed, J. A., Huang, W., Nallapareddy, S. R., Teng, F. \& Murray, B. E. (2004). Influence of origin of isolates, especially endocarditis isolates, and various genes on biofilm formation by Enterococcus faecalis. Infect Immun $\mathbf{7 2}$, 3658-3663.

Murray, B. E., Singh, K. V., Ross, R. P., Heath, J. D., Dunny, G. M. \& Weinstock, G. M. (1993). Generation of restriction map of Enterococcus faecalis OG1 and investigation of growth requirements and regions encoding biosynthetic function. J Bacteriol 175, 5216-5223.

Nakayama, J., Kariyama, R. \& Kumon, H. (2002). Description of a 23.9-kilobase chromosomal deletion containing a region encoding $f_{s r}$ genes which mainly determines the gelatinase-negative phenotype of clinical isolates of Enterococcus faecalis in urine. Appl Environ Microbiol 68, 3152-3155.
Qin, X., Singh, K. V., Xu, Y., Weinstock, G. M. \& Murray, B. E. (1998). Effect of disruption of a gene encoding an autolysin of Enterococcus faecalis OG1RF. Antimicrob Agents Chemother 42, 2883-2888.

Qin, X., Singh, K. V., Weinstock, G. M. \& Murray, B. E. (2000). Effects of Enterococcus faecalis $f_{s} r$ genes on production of gelatinase and a serine protease and virulence. Infect Immun 68, 2579-2586.

Qin, X., Singh, K. V., Weinstock, G. M. \& Murray, B. E. (2001). Characterization of $f_{s} r$, a regulator controlling expression of gelatinase and serine protease in Enterococcus faecalis OG1RF. J Bacteriol 183, 3372-3382.

Roberts, J. C., Singh, K. V., Okhuysen, P. C. \& Murray, B. E. (2004). Molecular epidemiology of the $f s r$ locus and of gelatinase production among different subsets of Enterococcus faecalis isolates. J Clin Microbiol 42, 2317-2320.

Shockman, G. D. \& Cheney, M. C. (1969). Autolytic enzyme system of Streptococcus faecalis. V. Nature of the autolysin-cell wall complex and its relationship to properties of the autolytic enzyme of Streptococcus faecalis. J Bacteriol 98, 1199-1207.

Sifri, C. D., Mylonakis, E., Singh, K. V., Qin, X., Garsin, D. A., Murray, B. E., Ausubel, F. M. \& Calderwood, S. B. (2002). Virulence effect of Enterococcus faecalis protease genes and the quorum-sensing locus $f_{s} r$ in Caenorhabditis elegans and mice. Infect Immun 70,

5647-5650.

Singh, K. V., Nallapareddy, S. R., Nannini, E. C. \& Murray, B. E. (2005). Fsr-independent production of protease(s) may explain the lack of attenuation of an Enterococcus faecalis $f_{s r}$ mutant versus a gelE-sprE mutant in induction of endocarditis. Infect Immun 73, 4888-4894.

Waters, C. M., Antiporta, M. H., Murray, B. E. \& Dunny, G. M. (2003). Role of the Enterococcus faecalis GelE protease in determination of cellular chain length, supernatant pheromone levels, and degradation of fibrin and misfolded surface proteins. J Bacteriol 185, 3613-3623. 